Estudios Románicos, Volumen 28, 2019, pp. 191-203

ISSN: 0210-491

eISSN: 1989-614X

DOI: https://doi.org/10.6018/ER/382311

\title{
INSURRECTIONS POPULAIRES ET DÉMYSTIFICATION D'UN ORDRE SOCIOPOLITIQUE ALTERNATIF DANS DEUX FICTIONS
}

(Popular insurrection and demystification of an alternative sociopolitical order in two fictions)

\author{
Pierre Suzanne Eyenga Onana* \\ Université de Yaoundé I (Cameroun)
}

\begin{abstract}
A study of Nathalie Etoke's Je vois du soleil dans tes yeux and Francis Bebey's La Poupée Ashanti reveals that beyond the status of anecdotal novels that defines them, it is, in depth, the postulation of a political change that mobilizes the two novelists in the depiction of a corrupt society. But what is the genesis of these popular movements? How and why are they literally represented? How, in fine, are political tensions managed by the local power and what aspirations then feed the actors in "order of battle» in the deciphered novels? To answer to this question, we use the sociocritical framework as implemented by Pierre Barbéris and Henri Mitterand. On that basis, we read novels throughout a triadic work plan. We first question the nature of social unrest to show that the novel does not limit itself to expressing a meaning that is already there. Then, we prove that through the work of writing, it modifies the previous equilibrium of meaning so as finally to refract and transform all at once, the social discourse in order to promote the living together policy.
\end{abstract}

Keywords: Political change; Sociocriticism; Demythization; Popular insurrections; Living together policy.

Résumé : Une lecture de Je vois du soleil dans tes yeux de Nathalie Etoke et La Poupée Ashanti de Francis Bebey révèle qu'au-delà du statut de romans anecdotiques qui les définit, c'est en profondeur la postulation d'un changement politique qui mobilise les

${ }^{*}$ Adresse pour la correspondance : Pierre Suzanne Eyenga Onana, Université de Yaoundé I (Cameroun), URFD Langues et Littératures, Département de Littérature et Civilisations Africaines, S/C Père Rigobert Touye, Paroisse de l'omnisports, BP 185/C-451 Yaoundé-Cameroun [eyonapiers@gmail.com]. 
deux romanciers dans la description d'une société corrompue. Mais quelle est la genèse de ces mouvements populaires ? Comment et pourquoi sont-ils littéralement représentés ? Comment, enfin, les tensions politiques sont-elles gérées par le pouvoir local et quelles aspirations nourrissent alors les acteurs en «ordre de bataille» dans les romans décryptés ? Pour répondre à cette interrogation, nous utilisons le référentiel sociocritique théorisé par Pierre Barbéris et Henri Mitterand. Sur cette base, nous adoptons un plan triadique. Nous questionnons d'abord la nature du mal social pour montrer que le roman ne se limite pas à exprimer un sens déjà là. Ensuite, nous prouvons que, par le biais du travail d'écriture, il modifie l'équilibre antérieur du sens pour finalement réfracter et transformer, tout à la fois, le discours social afin de postuler la politique du vivre ensemble.

Mots clés : Changement politique ; Sociocritique ; Démythisation ; Insurrections populaires; Vivre ensemble.

\section{Introduction}

L'avènement récurrent en Occident (Vénézuela ; France ; Chine), et en Afrique des révoltes multiformes (Algérie, Soudan du Sud), sonne le glas du monopole étatique. Avec son corollaire, le musellement des populations sous la bannière de dictatures parfois sournoises mais pourtant très sanglantes, on assiste dans certaines régions du monde au secouement, au démantèlement voire au déracinement pur et simple de régimes politiques autocratiques solidement implantés, à l'instar des États du Maghreb (Tunisie, Égypte et Lybie). Si l'Afrique au Sud du Sahara n'est guère épargnée par cette vague de mouvements insurrectionnels, tel que celui enregistré tout récemment au Burkina Faso, ainsi que les récriminations populaires inhérentes aux crises postélectorales diverses (Gabon, Tchad), c'est dire à quel point la thématique de l'insurrection se révèle d'une actualité obsédante. Aussi mérite-t-elle une réflexion approfondie en amont comme en aval. Dès lors, il convient de s'interroger sur la représentation littéraire des mouvements sociaux en questionnant les mobiles qui les soustendent depuis leur genèse jusqu'à leur déploiement sociétal. On se demande alors comment ces tensions revendicatives sociopolitiques sont à la fois appréhendées et gérées par le pouvoir local. On s'intéresse aux aspirations des acteurs contestataires dans la perspective de sonder la portée éthique des mouvements d'humeur en question.

Le référentiel convoqué pour répondre à ce questionnement est la sociocritique théorisée par Pierre Barbéris et Henri Mitterand. Pour le premier, la grille de lecture sociocritique fédère plusieurs domaines des sciences humaines. Elle s'opère « à partir d'une recherche et d'un effort tâtonnant et découvreur qui invente un nouveau langage, fait apparaître de nouveaux problèmes et pose de nouvelles questions » (Barbéris 1990 : 122). Dans la démarche d'Henri Mitterand, «tout roman propose à son lecteur, d'un même mouvement, le plaisir du récit de fiction, et, tantôt de manière explicite, tantôt de manière implicite, un discours sur le monde » (1980:5). Il apparait, en d'autres termes, que le récit déploie un double enjeu, à la fois reproductif (la mimésis), et productif (la sémiosis). C'est en raison de cela que Mitterand entrevoit en filigrane trois étapes clefs dans le décryptage d'un roman. Il soutient ainsi que : « le texte de roman ne se limite 
pas à exprimer un sens déjà là ; par le travail de l'écriture, il produit un autre sens, il modifie l'équilibre antérieur du sens ; il réfracte et transforme tout à la fois, le discours social» (Mitterand $1980: 7$ ).

Avant de procéder à l'analyse du corpus, quelques précisions s'imposent. Je vois du soleil dans tes yeux de Nathalie Etoke raconte l'histoire de Wélisanè qui se résout à qui se résout à faire chemin avec Valérie, son amie, dans la quête de l'homme blanc providentiel qui les sortira de la misère. Au hasard d'une sortie nocturne, elle rencontre Jean-Marc (Ruben), le leader de la Jeunesse Africaine en Colère (JAEC). Ce dernier se bat pour l'avènement d'une Afrique nouvelle. Confrontés aux vicissitudes de la vie politique, tous deux mobilisent la jeunesse rebelle en vue de changer le cours de l'histoire de l'Afrique. Dans La Poupée Ashanti, Francis Bebey met en scène une jeune orpheline nommée Edna, qui incarne et protège les intérêts des vendeuses du marché après son viol par Bunéfo, l'instituteur de son école. Pour une raison politique liée à l'arbitraire, l'une d'elles, madame Amiofi, se voit retirer le permis de vendre au marché. Cette grave injustice entraîne une marche populaire des femmes du marché vers le Parlement, sous la forme d'une révolte militante visant à faire germer l'idée de la démocratie dans les esprits.

\section{Modalisations des querelles sociales}

Par mouvements sociaux, il faut entendre toutes formes d'action collective qui revendiquent, dans la dynamique de rapports de force entre protagonistes, l'accès aux ressources, à la justice sociale, à la liberté politique et à l'égalité des chances au plus grand nombre, voire à tous. (Cefaï 2007 : 49). Les formes d'engagement ou d'action collective trouvent de ce point de vue un terreau fécond dans les partis politiques, les classes sociales diverses, les syndicats, les réseaux sociaux, les groupes informels ou spontanés, pour ne citer que ces quelques modalisations. Les acteurs sociaux impliqués dans la revendication sociale forment la "société civile" et négocient des ressources et des attributs identitaires (Edwards 2009 : 67).

Dans Je vois du soleil dans tes yeux, mobilisée autour d'un parti de rassemblement, la JAEC, la « Jeunesse Africaine en Colère » (Etoke 2008 : 34), se dresse contre des institutions étatiques truquées qui occultent les droits de l'homme et ignorent une partie importante du peuple. Les lexèmes empreints d'injonction que convoque le leader de ces jeunes pour susciter en eux le goût pour la revendication sont évocateurs : « il faut que cela cesse. Mes frères, mes sœurs, il est grand temps de se prendre en main. Venez participer à la grande lutte pour le changement. Il ne viendra pas d'ailleurs, il ne viendra pas des autres. » (2008: 34). Le jour de la manifestation est le point culminant de l'activité revendicatrice des jeunes. Instance de subversion comme le dit Lucien Goldmann, le roman devient alors « l'histoire d'une recherche dégradée [...] de valeurs authentiques dans un monde dégradé lui aussi » (1964: 23). Les leviers de cette dégradation sont activés lorsqu'au cours du défilé populaire les jeunes brandissent des pancartes en scandant des slogans récriminateurs qui paraphrasent parfois les grands hommes de l'histoire mondiale tels que Karl Marx : « le peuple [...] fait grève. [...] Jeunes Africains de tous Pays, Unissez-Vous » (Etoke 2008 : 58). 
À l'instar des jeunes dans Je vois du soleil dans tes yeux, les femmes du marché se rassemblent dans le cadre de leur association. Selon Mam, celle-ci « forme le groupe syndical le plus important » du pays. (Bebey 1973 : 28). Elles y paient leur cotisation et se mobilisent pour soutenir l'une d'elles, Mme Amiofi, qui connaît des frustrations d'ordre professionnel parce que sa fille a une connexion sentimentale avec un député de l'opposition. Il convient avant toute chose de disséquer les figures sociales qui impulsent le mouvement insurrectionnel dans le corpus.

\subsection{Des figures de la contestation populaire : entre rupture, permanence et spécificités}

Les figures sociales qui crient leur désarroi aux gouvernements sont en priorité les femmes et les jeunes. Contrairement à des personnages instruits tels que Wélisanè dans Je vois du soleil dans tes yeux, ou encore Esther dans Le Journal intime d'une épouse de Bonono, Francis Bebey choisit dans La Poupée Ashanti de mettre en scène un personnage atypique : la femme du marché. Son trait identitaire de base est l'analphabétisme. Mam, la grand-mère d'Edna, lui rappelle ainsi son aversion pour cette institution : «Toi, fille de mon sein, je crois que c'est une bonne chose que tu ne sois pas restée trop longtemps à l'école. Car avec toute l'intelligence que tu as dans le crâne, où donc irais-je vivre si tu y mettais la science des Blancs?» (Bebey 1973 : 27). Pour elle, la femme est prédestinée aux considérations essentialistes de la vie : " elle naît femme », elle ne le devient pas, pour parodier autrement Simone de Beauvoir (1949 : 13). Selon cette vision essentialiste, telle que l'entrevoit Calixthe Beyala dans Lettre d'une Africaine à ses scurs occidentales, la femme est " née à genoux aux pieds de l'homme (1995:11)». Toutes choses qui en illustrent la réification. Mais Bebey entend mettre en scène une bâtarde battante pour montrer que les rôles sociaux ne devraient pas toujours être conditionnés par le sexe des individus. Il contredit ainsi Mam qui affirme à l'adresse d'Edna qu' " écrire [...] c'est l'affaire de ces femmes qui veulent travailler dans les bureaux, comme si elles étaient des hommes. Toi, tu n'as pas besoin de cela. Et d'ailleurs, tu sais bien que le ciel, lui non plus, n'a pas attendu cela de toi. » (Bebey 1973 : 27).

Le même élan de révolte qui anime les femmes du marché, sous la bannière d'Edna dans La Poupée Ashanti, secoue la jeunesse frustrée du Koumkana, sous les auspices de Wélisanè et Jean-Marc dans Je vois du soleil dans tes yeux. À première vue ladite révolte n'en donne pas l'air. En ce sens, on peut affirmer que « toute mutation en voie de se faire reste, pendant une période variable, masquée ; elle est d'abord noyée dans les configurations latentes dont toute société est porteuse, destinées les unes à façonner l'avenir, les autres à disparaître sans se réaliser » (Balandier 1970 : 25). Les mobiles qui sous-tendent la colère des de l'association des femmes du marché et la JAEC sont nombreux. Ils vont du règne de la démagogie au triomphe d'un népotisme outrageant.

\subsection{Les ressorts d'un dysfonctionnement gouvernemental systématisé}

Népotisme et démagogie constituent quelques-uns des leviers sociaux qui définissent les contours de l'inertie dans la gestion gouvernementale. Celle-ci est « proba- 
blement la conséquence de la tactique qui consiste à laisser des questions fondamentales longtemps irrésolues dans l'espace public » (Olinga 2009 : 51). Bien que figurant comme groupe syndical le plus influent du pays, l'Association des femmes du marché fait les frais de la démagogie présidentielle. "Présentent dans toutes les activités du pays » (Bebey 1973 : 28), les femmes du marché peuvent infléchir la donne électorale en orientant à guise l'issue d'une élection. Dénonçant la veine démagogique dans laquelle baigne désormais le gouvernement du Docteur, elles ont en travers de leur gorge le plébiscite à lui accordé en vue de son accession à la magistrature suprême. Pour Mam, « le Docteur n'a pas fait grand-chose pour rappeler aux femmes du marché qu'il leur est reconnaissant de l'action qu'elles ont menée en sa faveur » (Bebey 1973 : 28). Ce qui signifie que le chef de l'État en accusation dénie la méritocratie et voue un culte au favoritisme.

Dans la même veine, la jeunesse du Koumkana par la voix du tribun de la plèbe récrimine dans Je vois du soleil dans tes yeux pour des motifs quasi similaires. Estimant le Koumkana en ruine à cause de la mainmise de " dirigeants de pacotille » (Etoke 2008 : 33), le tribun pointe du doigt l'inégale répartition des biens entre citoyens et ajoute à la décharge desdits dirigeants que : « sans scrupule, ils plastronnent dans leurs costumes, construisent des châteaux, vont en villégiature en Europe et [...] laissent crever [le peuple] » (2008 : 33). Comme relevé dans La Poupée Ashanti, la vague du népotisme touche avantageusement les membres du cercle familial du chef de l'État koumkanais au détriment du petit peuple et de sa progéniture. Celle-ci passe la " journée à fouiller dans les poubelles de la survie » (2008: 34$)$, à défaut de périr, purement et simplement. Quoiqu'issu de l'entourage présidentiel, Jean-Marc n'en combat pas moins les frasques népotiques en confiant : « Mon père est le cousin germain du président, ce qui lui vaut sa permanence au gouvernement depuis une vingtaine d'années » (2008: 78). Qui pis est, il récuse les prébendes auxquelles a droit son géniteur : « Il sert la Nation et se sert au passage. Vacances sur la croisette ou aux Bahamas, appartement sur les Champs Elysées, château au village, parc automobile hors du commun, soins médicaux dans l'abondance, l'excès de gaspillage » (2008: 78).

De même, en accordant des avantages immérités aux incapables, le Docteur dans $L a$ Poupée Ashanti priorise l'ascension professionnelle des membres de sa famille et celle de son entourage dans la hiérarchie qu'il dirige, au détriment du mérite et de l'intérêt général. Mam relève avec dépit pour les déplorer ces faillites qui néantisent la femme du marché : «il n'a songé à faire entrer dans son gouvernement que des membres de sa famille, ou des gens de son parti. C'est cela, la politique, fille de mon sein, que veux-tu y faire ? » (Bebey 1973 : 29). Ce culte de sa famille secrète aussi chez le Docteur un autre vice : le narcissisme et le monopole étatique.

Définissant le concept d'inertie qui n'a de cesse de prendre possession de la cité africaine, Olinga affirme qu'elle se décline en trois pôles : "d'abord le cynisme déterminé et l'égoïsme forcené de quelques-uns puissamment organisés ; ensuite le résignation et l'indifférence du plus grand nombre, [...] ; enfin l'impuissance, le découragement et l'inorganisation des hommes de bonne volonté » (Olinga 2009 : 10-11). La forme d'inertie caractéristique de la posture du Docteur est le culte de sa personnalité. Celui- 
ci se manifeste à travers le matraquage. étatique et la surmédiatisation des sorties du chef de l'État. Il s'agit de stratégies mises en place par le pouvoir pour contourner la vérité et détourner le peuple de la réalité observée sur le terrain au moyen de la flagornerie voire de la duperie pure et simple. Mam juge disharmonieux l'usage fait d'un bien public comme la radio du moment où, dans les propos suspects diffusés à l'adresse du peuple, s'entremêlent mensonge et publicité en faveur d'un individu élu supposé se mettre au service de la population. Par le biais d'un discours itératif qui ressasse des faillites dans la gestion par l'Etat des ressources humaines, Man relève pour le déplorer : «à chaque fois, on te dit que Le Docteur a fait quelque chose pour le pays, et puis, le lendemain, [...]. Toute l'année, c'est du Docteur que l'on parle, toujours du même. En fin de compte, je ne vois pas ce qu'il a fait pour le pays » (Bebey 1973: 68). Son laxisme sidérant plonge le pays dans une crise de l'État de droit nous invitant à interroger le style des romanciers dans la diction du vice social.

\section{De l'esthétique à la portée éthique de la syntaxe des mouvements sociaux}

Pour Henri Mitterand, le roman signifie également par le travail de l'écriture qui lui donne de produire un autre sens en modifiant toutefois l'équilibre antérieur du sens. On peut dès lors convenir qu' « avec des mots, l'écriture crée un sens que les mots n'ont pas au départ » (Barthes $1981: 15)$. Cette antépénultième partie du travail est consacrée à l'esthétique des romanciers dans l'analyse faite des mouvements sociaux. À la relecture du corpus, un certain nombre de stratégies d'écriture entre en résonnance avec le message des romanciers sur la question des mouvements sociaux. L'on évoquera l'ironie comme figure de rhétorique, aux côtés d'autres faits d'écriture que sont l'intertextualité et l'inter-généricité.

\subsection{La stylisation des maux sociaux}

S'agissant de l'ironie, elle se définit comme « un énoncé par lequel on dit autre chose que ce que l'on pense en faisant comprendre autre chose que ce que l'on dit. Il fonctionne comme subversion du discours de l'autre [...], et qui exprime implicitement le désaccord de l'énonciateur » (Lejeune 1980 : 24-25). Parce qu'illettrée, Mam produit un discours ironique à la limite de l'insensé à l'adresse de ses principales interlocutrices que sont Edna et Princess dans La Poupée Ashanti. En militant pour l'expression des libertés, elle appelle de tous ses vœux et ceci sans le savoir, l'avènement de la démocratie. Or, qui dit démocratie dit opposition et milite donc en faveur de la multiplication des idées politiques. Pourtant, à la suite de l'arrestation d'un député de l'opposition, Mam confie à Princess que : « il faut un seul parti, un parti unique dans ce pays, car plus il y aurait de partis, moins vite nous pourrions progresser. Après tout, l'opposition n'avait qu'à gagner la bataille pour la libération nationale » (Bebey 1973 : 74).

Dans le prolongement de cette occurrence ironique, il faut également mentionner une autre qui se fonde cette fois sur l'interrogation rhétorique. Encore appelée « question rhétorique » ou " question de style », cette figure de pensée n'appelle même pas 
de réponse, tant la réaction attendue est considérée, même de manière forcée, comme évidente (Robrieux, 2000 : 116). Cette figure se lit dans La Poupée Ashanti lorsque Mam se méprend sur le vrai sens du mot démocratie. Pourtant, sans l'existence d'une quelconque opposition, il serait inopportun de parler de démocratie. Se demandant alors si l'opposition sert la cause de la démocratie dans un pays en quête de paix, Mam se perd en conjectures : « Et je te demande : pourquoi sont-ils dans l'opposition? n'ont-ils pas encore compris qu'il faut s'unir pour construire la nation ?» (1973: 74). Or, sans une opposition solide et des opposants dans un pays, la vie politique devient monocorde et le manque de cette diversité politique entraine le diktat. Voilà d'ailleurs pourquoi il est paradoxal pour Mam de croire que les opposants sont des ennemis pour le pays lorsqu'elle souligne à leur charge qu'ils « s'acharnent à démolir ce que fait le gouvernement, jusqu'au jour où tout s'écroule, par leur faute [...] Mais qui sait si leur parti est vraiment le bon?» (1973: 74). Pour Robrieux, le paradoxe naît du fait que cette figure de rhétorique choque le sens commun par l'association de termes contradictoires dans un énoncé prédicatif (2000: 90 ).

Toute la critique formulée en supra à l'endroit de l'opposition s'opère au moyen de la figure de l'hypotypose. Il s'agit d'une « description ou d'un récit qui permettent au lecteur de se représenter [...] une scène, comme s'ils étaient sous ses yeux » (Robrieux 2000 : 116). L'entrée d'Edna au Parlement pour y plaider la cause d'Amiofi est un cas illustratif : «Edna arracha son foulard de la tête, et se mit à le faire tourner dans l'air en marchant courageusement vers l'entrée du Parlement. [...]. Pourtant Edna ne voulut pas se retourner, pas même pour voir si d'autres personnes, hommes ou femmes, la suivaient » (Bebey 1973 : 101).

Bien plus, l'un des traits les plus marquants de l'écriture d'Etoke réside dans sa polyphonie. À la fois intertextuelle et multiculturelle, cette écriture ne saurait manquer d'interpeller le critique sur les enjeux sémantiques qui l'articulent. S'agissant de l'intertextualité qui y est inscrite, elle renvoie à la " présence littérale (plus ou moins littérale, intégrale ou non) d'un texte dans un autre : la citation, c'est-à-dire la convocation explicite d'un texte, à la fois présenté et distancé par des guillemets, est l'exemple le plus évident de ce type de fonctions ; qui en comporte bien d'autres » (Genette 1979 : 87). Fondée sur l'étude de l'intertexte, l'intertextualité désigne, de plus, l'ensemble des textes mis en relation par le biais de l'allusion, du plagiat, de la référence et du lien hypertexte dans un texte donné. Mais il convient de souligner que loin de faire l'objet de plagiat dans l'imaginaire d'Etoke, l'intertextualité s'inscrit davantage dans une dynamique d'exposition des faits qui accablent les dirigeants du Koumkana et leurs pratiques inopérantes face aux populations dont ils ont la charge.

La crise des libertés que flétrit la jeunesse est en outre évoquée lorsque la romancière emprunte à dessein au discours politique des stars de la chanson mondiale telles que le Nigérian Fela Ransome Kuti. Les propos de ce dernier sont révélateurs de la profondeur du mal social qui n'a de cesse de gangrener la société politique. Dans un propos provocateur articulé autour de la rhétorique de la néologie, on peut désormais entendre la rue grogner en ces termes à travers un mélange manichéen campé par une langue qui choquerait les destinateurs du discours : « Movement of the People Democracy, 
yes ! demonicracy, no ! politic, yes ! Polytricks, no ! In the words of Fela Ransome Huti M.O.P, Movement of the People Democracy, yes : Demonicracy, no ! Politic, yes ! Polytricks, no ! [Mouvement Démocratique pour le Peuple, oui : Démoncrarcie, non ! Politique, oui! Politriches, non! ]» (2008:58). Si ces mots évacuent toute tendance à la systématisation de la " démoncratie », en tant qu'elle se positionne comme une idéologie politique déstabilisatrice, s'ils invitent au contraire à valider l'idée de la démocratie entrevue comme modèle politique alternatif, c'est parce que ce modèle politique porte haut l'étendard des droits humains en exaltant les libertés. L'exhortation au courage et à la persévérance au combat militant donne droit aux citations in extenso de références connues. Le psaume 23 de la Bible autant que la célèbre formule de Birago Diop « les morts ne sont pas morts » (2008: 113) en sont des cas illustratifs. Par ailleurs, la misère au cœur de laquelle croupissent les populations est mise au jour au moyen de l'allusion littéraire, forme intertextuelle moins explicite et moins littérale dont le référent renvoie à un texte-source. Tel est le cas du roman Ville cruelle d'Eza Boto, référé dans Je vois le soleil dans tes yeux lorsque le tribun, dans un registre populaire, médite sur les conditions de vie quotidiennes pitoyables au sein du quartier S'en-fou-la-mort : « il faut que les gens de la haute viennent faire un tour ici. Hé ben, Tanga Nord et Tanga Sud existent toujours. Il faut je relise Ville cruelle » (2008: 111).

L'usage de mots-valises est également l'une des marques d'écriture relevées dans Je vois le soleil dans tes yeux. Il s'agit de néologismes qui font l'amalgame de deux mots (Robrieux 2000 : 64). Cette figure de mot est notée quand le tribun récuse les maux auxquels s'expose au quotidien la jeunesse du Koumkana : ventrocrate ; mangeocratie ; fusilcratie ; ventrocratie $(2008: 34 ; 58)$. On pourrait à travers ces occurrences lire une combinatoire opérante entre les noms " ventre et fusil », le verbe " manger », et les suffixes «crate » et «cratie ", à l'instar des mots «bureaucrate » ou encore " démocratie ». La romancière stigmatise alors l'option vicieuse consistant pour les dirigeants à faire détourner les biens communs pour la satisfaction de leurs besoins égocentriques. Il s'agit aussi pour Etoke de mettre à l'index le vilain désir de faire de la barbarie un mode de communication au sein duquel le fusil devient le point de chute d'une philosophie existentielle caduque dont la finalité reste le confinement du peuple au silence.

Toute cette trame intertextuelle est négociée à travers la mise en exergue de plusieurs genres littéraires. C'est ainsi que genres poétique, dramatique, épistolaire et musical s'imbriquent harmonieusement dans les deux fictions décryptées, pour mettre en lumière la dimension interdisciplinaire très souvent caractéristique du roman africain contemporain. On peut évoquer ce poème de Werewere Liking allusivement déclamé par Ruben dans la perspective de dévoiler, en filigrane, l'identité des nouveaux Africains qui naitront des cendres de ceux qui se seront battus : «Ah! mes enfants .../Ils ne seront pas les fils des colons [...]/ Ils ne seront plus les fils de l'assujetti/[...]Ils seront libres et forts et beaux/Ils seront de jaspe et de corail » (2008 : 169). En fin, par le biais de la citation de l'hymne national sud-africain, la romancière implore la bénédiction de Dieu sur ses fils en supprimant partout guerre et souffrance lwayo : «Nkosisikelel'iAfrika / MaluphakamupondoLwayo /Yivaimitandazoyetu/ Nkosisikelelathinalusapholwayo / 
YithlaMoyaYthaMoya » (2008 : 191). Bien plus, le roman d'Etoke est rédigé dans plusieurs langues. Le français et l'anglais se voient ainsi ponctuées par des langues nationales ou locales telles que le pidgin ou le douala, pour renforcer l'ancrage culturel africain de la romancière : «A muna o é/A muna o é /O s'éyapè/ Kakamulémabènguinya [mon enfant ne pleure plus ; serre ton cœur sois forte]» (2008: 126).

L'analyse des occurrences esthétiques qui précède permet dans un premier temps d'affirmer le caractère transculturel des fictions disséquées comme pour dire que « toute œuvre littéraire a ses traits individuels ; mais elle partage aussi certaines propriétés avec d'autres auvres » (Welleck ; Warren 1971 : 22). C'est l'ouverture de la littérature à d'autres univers culturels qui autorise à en affirmer la pluralité d'une œuvre à une autre. On peut dès lors partager l'avis d'un critique connu lorsqu'il soutient qu' « interpréter un texte ce n'est pas lui donner un sens (plus ou moins fondé, plus ou moins libre) c'est au contraire apprécier de quel pluriel il est fait » (Barthes $1970: 11$ ). Aussi, convient-il à cet égard d'examiner à présent la portée sémantique et/ou la dimension éthique qui traverse d'un bout à l'autre les textes en examen.

\subsection{Portée éthique des mouvements sociaux}

À en croire Gaëtan Picon, «l'œuvre d'art - et singulièrement l'œuvre littéraire - [...] s'offre à l'esprit comme objet d'interrogation, [...] Dès qu'elle rencontre un regard, [elle] appelle irrésistiblement la conscience critique : celle-ci l'accompagne comme l'ombre suit chacun de nos pas » (Picon 1953 : 11). Partant de ce point de vue, on observe que les jeunes qui s'activent au péril de leur vie dans le cadre de l'Association de femmes du marché ou de la JAEC, donnent le témoignage d'une gouvernance problematique. Celle-ci se montre radicale mais aussi et surtout radicale parce que radicale et verticale dans la mise en pratique de ses stratégies managériales. Népotique, elle se caractérise par l'autoritarisme tandis que la violence alimente régulièrement les contradictions fonctionnelles qui le sous-tendent. Toutes choses qui fondent l'argument que « ne se reconnaissant [...] pas dans les décisions prises sans leur participation, relativement à la définition des préférences collectives, les citoyens ont tendance à se rebeller contre cette gouvernance qui fait cyniquement l'impasse sur leur citoyenneté » (Ayissi 2003 : 264).

Mais l'option pour la violence ne se révèle pas un choix opérant pour un retour au calme des esprits surchauffés. Au contraire, la négociation et le débat constructif s'offrent comme importants leviers pour conjurer les démons de la violence. Dans un roman comme dans l'autre, la force des manifestants est telle que rien ne peut les contenir si ce n'est la violence étatique, preuve que l'État gagnerait à amorcer le dialogue nécessaire pour désamorcer la crise lorsqu'elle sévit. Dans La Poupée Ashanti, le narrateur relève ce manquement en affichant la posture contestable des policiers manœuvrant péniblement pour pouvoir arrêter la marche : «les policiers eurent alors recours à toutes sortes d'engins de mort, dont la stupidité n'enlevait malheureusement rien à l'efficacité. De nombreux coups de feu partirent, dirigés cette fois contre les manifestants dont la témérité avait fini par exaspérer les représentants de l'ordre » (Bebey 1973 : 102). De même, dans Je vois du soleil dans tes yeux, les jeunes qui se retrouvent au stade des Trois 
Voleurs le jour de la manifestation s'érigent en véritable obstacle insurmontable sur la voie des politiciens aux fins de défier le système politique cavernicole qui les réifie. Leur credo est le suivant : " arrêtons de nous asseoir et d'attendre ! Bougeons! » (Etoke $2008: 60)$.

Au regard de ce qui précède, il importe pour le Koumkana de cesser d'apparaître comme cet espace des damnés où la violence passe pour être la règle. Il doit rompre avec l'escalade de la violence institutionnalisée en promouvant des contextes d'action réfractaires à l'implosion dans les mentalités chez les laissés-pour-compte. La revendication populaire utile se révèle un moyen de communication opportun qui gagnerait à s'inscrire dans les mœurs. Son but ne saurait de ce point de vue être de combattre la machine étatique mais de l'aider à mieux prendre en charge les récriminations des populations. $\mathrm{Au}$ Koumkana pourtant, règne en effet une telle tradition de violence et d'intimidation que toute entreprise révolutionnaire est vue d'un très mauvais œil puisque les instigateurs sont aussitôt réduits au silence ainsi que le souligne la narratrice omnisciente : « j'oubliais que j'étais au Koumkana un pays où toutes les têtes qui dépassent sont coupées, tranchées au couteau d'une démocratie aux relents de dictature » (2008:60). D'ailleurs, outre la tactique d'intimidation collective relevée plus haut, le gouvernement du Koumkana a plus d'un tour dans son système de répression pour réduire au silence tous ceux qui, à l'instar de Ruben, lèvent la tête pour le défier. Le lavage de cerveau fait partie de ses multiples stratégies de néantisation des « rebelles ». Victime lors de la manifestation d'un enlèvement perpétré par l'armée gouvernementale, Jean-Marc, le leader des jeunes, est conduit à un endroit tenu secret : « impossible d'aller demander de l'aide à qui que ce soit. Ici, police et justice appartiennent aux gens d'en haut. Impossible de consulter sa famille» $(2008: 135)$.

Mais on le sait, la volonté d'avancer malgré tout compte plus que tout lorsque le souci des hommes à se réaliser eux-mêmes prédomine sur les violences à subir. Autrement dit, " celui qui veut le progrès ne prête aucune attention aux étiquettes qu'on peut lui coller sur le dos avec le dessein manifeste de le décourager et de l'intimider » (Njoh Mouelle 1980 : 59). À cet égard, la nouvelle jeunesse postulée par les romanciers à l'aube du IIIème millénaire dévoile le visage d'une race de guerriers pacifiques, plus volontaires quant au choix de l'angle d'attaque adopté dans la perspective de se libérer de l'hydre de la mal gouvernance qui l'enserre. Il s'agit dans un premier temps d'exorciser le mal multiforme qui mine la société en vue de le conjurer résolument. Ces maux ont pour noms : la faillite de l'hospitalité dans les hôpitaux, le monopole étatique, le népotisme, la corruption, le chômage, la crise des libertés, la manipulation de la jeunesse, la violence à outrance, pour ne mentionner que ces occurrences romanesques. Il s'agit alors pour les jeunes réformateurs de porter eux-mêmes le changement en faisant tonner leurs voix afin que naissent de nouveaux espoirs pour la jeunesse, fer de lance de toute nation futuriste.

Dans cette perspective, il s'agit de renforcer le combat que mènent Edna et les femmes du marché, de même que Jean-Marc et Wélisanè, afin que naisse enfin un jour neuf : " une Afrique meilleure, une Afrique nouvelle ; une Afrique différente, une Afrique sans famine, une Afrique sans guerres, une Afrique sans dictateurs, une Afrique sans 
pilleurs, une Afrique de Liberté et de droits de l'homme » (2008: 83). Autant dire que la construction de l'Afrique de demain commence par le grand rêve d'aujourd'hui. Si apparemment elle semble une « mission impossible pour l'instant ..., [la jeunesse] pouvai[t] néanmoins la Rêver... Ils [les tortionnaires] ne [l'] empêcheront pas de Rêver. Ils ne briseront pas les ailes de [s]es pensées chimériques » (2008:189).

Par ailleurs, la signature qu'accorde le ministre des finances à Amiofi pour lui restituer son permis de vendre au marché consacre la victoire de la raison sur la déraison et le trafic d'influence pompeux. Elle témoigne du fait que les revendications utiles, sous forme de grève ou de marches populaires ou collectives, sont parfois nécessaires, déterminantes voire décisives pour faire sauter certains verrous qui hypothèquent l'éclosion de la paix ou tout au moins en obèrent son avènement dans la cité des hommes. Comme le souligne fort opportunément Patrice Nganang, « qu'elle soit de libération ou civile, la guerre est toujours un échec de l'imagination - l'histoire de l'Afrique et du monde sont là pour nous l'apprendre - ; l'écriture au contraire en est un triomphe » $(2005: 25)$. Ce postulat s'applique bien à notre corpus au regard du manque de compréhension dont font montre les autorités en charge du devenir des populations. Elles choisissent plus souvent la voie de la contradiction, de la violence et du crime organisé, plutôt que celle du dialogue ou de la négociation concertée. Ainsi, si le lavage de cerveau dont est victime Ruben se révèle une étape stratégique dans la lutte des jeunes militants au sein de la JAEC, force est d'affirmer que le choix du gouvernement renforce au plus haut point l'action de ce réformateur. Désormais, il communique dans la plus grande liberté par le biais de chants et de poèmes qui séduisent de plus en plus d'adeptes au fil des jours. Comme pour dire alors que le train de la bonne gouvernance transcende les obstacles physiques pour s'inscrire inexorablement dans l'ère du temps.

\section{Conclusion}

À tout prendre, l'ère du temps en question c'est celle des réformes pertinentes, efficientes et urgentes, celle des ajustements utilitaires et communautaires qui, seuls, démontreront que chaque composante sociologique devrait, dans son aire de compétence, contribuer à l'édification du développement holistique du pays. Autant reconnaître que moins que de simples moments d'activisme de mauvais aloi ou de turgescence sociale, les mouvements sociaux visent davantage à impulser une nouvelle dynamique gouvernementale incitative et alternative qui induirait chez les dirigeants la validation du postulat que pour gouverner le mieux possible et plus longuement, il conviendrait avant tout de gouverner le moins possible en collaborant à la promotion d'une citoyenneté participative. Bien plus, dans une cité organisée, les mouvements sociaux se révèlent un baromètre crédible et/ou fiable dans l'évaluation de l'état d'esprit dans lequel baigne le peuple en tant qu'indice de développement clef. Ils sont autant illustratifs du pourcentage d'épanouissement des populations que du degré de malaise qui les traverse. C'est en raison de cela qu'ils appellent en tout temps à un renouvellement des cadres juridiques et des perspectives gouvernementales dans le sens de laisser poindre une démocratie de proximité. C'est ce modèle étatique qui fédère et rapproche considérablement les admi- 
nistrateurs des administrés dans un processus de prise de décision collégial, celui qui les fait intervenir effectivement dans la gestion du devenir de la communauté. On est donc en droit de convenir avec un critique que « la littérature [...] paraît [...], la somme des réponses possibles aux questions réelles que se posent un homme et, à travers lui, une époque, une civilisation et, à la limite, l'humanité » (Doubrovsky 1966 : 93).

\section{BIBLIOGRAPHIE}

AYISSI, Lucien (2003) : " Gouvernance partagée et décentralisation ». Gouvernance partagée : la lutte contre la pauvreté et les exclusions. Yaoundé : CUP, 250-264.

BALANDIER, Georges (1970) : Sociologie des mutations. Paris : Anthropos.

BARBÉRIS, Pierre (1990) : «Sociocritique ». Introduction aux méthodes critiques pour l'analyse littéraire. Paris : Bordas, 121-153.

BARTHES, Roland (1970) : S/Z. Paris : Seuil.

BARTHES, Roland (1981) : Le Grain de voix. Paris : Seuil.

BEAUVOIR, Simone De (1949) : Le Deuxième sexe II. Paris : Gallimard.

BEBEY, Francis (1973) : La Poupée Ashanti. Yaoundé : CLÉ.

BEYALA, Calixthe (1995) : Lettre d'une Africaine à ses sæurs occidentales. Paris : Spengler.

CEFAÏ, Daniel (2007) : Pourquoi se mobilise-t-on? Les théories de l'action collective. Paris : La Découverte.

DOUBROVSKY, Serges (1966) : Pourquoi la nouvelle critique?. Paris : Mercure de France.

EDWARDS, Michael (2009): Civil Society. Cambridge: Polity Press.

ETOKE, Nathalie (2008) : Je vois du soleil dans tes yeux. Yaoundé : PUCAC.

GENETTE, Gérard (1979) : Introduction à l'architexte. Paris : Seuil.

GOLDMANN, Lucien (1964) : Pour une sociologie du roman. Paris : Gallimard.

LEJEUNE, Philippe (1980) : "Le récit d'enfance ironique : Vallès », Je est un autre. L'autobiographie de la littérature aux médias. Paris : Seuil, 24-25.

MITTERAND, Henri (1980): Le Discours du roman. Paris : Seuil.

NGANANG, Patrice (2005) : Le Principe dissident. Yaoundé : Interlignes.

NJOH MOUELLE, Ebenezer (ed.) (1980) : Développer la richesse humaine. Yaoundé : CLÉ.

OLINGA, Alain Didier (2009) : «L'intelligence de l'inertie ». Patrimoine. Yaoundé : CLÉ : 10-11.

PICON, Gaëtan (1953) : L'écrivain et son ombre. Paris : Gallimard.

ROBRIEUX, Jean-Jacques (2000) : Rhétorique et argumentation. Paris : Nathan. WELLEK, René; WARREN, Austin (1971) : La Théorie littéraire. Paris : Seuil. 


\section{PROFIL ACADÉMIQUE ET PROFESSIONNEL}

Pierre Suzanne Eyenga Onana est Maître de Conférences au Département de Littérature et Civilisations Africaines à la Faculté des Arts, Lettres et Sciences Humaines de l'Université de Yaoundé I (Cameroun). Ses recherches portent sur les modalisations littéraires du vivre ensemble, l'épistémologie de la littérature (sémiologie du texte littéraire africain et euro-américain écrit) ainsi que les questions de féminismes et les Gender Studies. Il est l'auteur d'une cinquantaine d'articles dont : « Le Nez sur la vitre d'Abdelkader Djemaï. Métaphore d'une enfance spoliée ou figuration d'une Algérie tourmentée ?», in Revue en ligne LICARC n5, L'Enfant dans la culture arabe contemporaine, Classiques Garnier, 2017, pp. 271-286 ; « Errance féminine et quête de survie : de la déconstruction des biais androcentriques à la légitimation de la fraternité universelle dans Femme infidèle de Tcha-Koura Sadamba ", in Revue en ligne ALETRIA, Brésil, Deslocamentos, Linhas de Fuga, Confins, n² 28, 2018, pp. 119-136 ; «De l'immigration clandestine à l'exil improvisée ; Une esthétisation du rêve hypothéqué dans Le Paradis du Nord de Jean Roger Essomba », in Revue en ligne Viaggatori, Italie, Deslocamentos, Linhas de Fuga, Confins, n 2, 2018, pp. 429-460 ; « Kama Kamanda et Tchikaya U'Tamsi : deux auteurs, deux allégories, une vision stylisée du fantastique et de l'étrange ", in Fantastique, étrange et merveilleux dans les productions francophones, Canada, Les Cahiers du GRELCEF, n 9, 2017, pp. 17-34 ; « Le viol, une variable du mal dans quelques romans camerounais », in Revue en ligne ALETRIA $27 \mathrm{n}^{\circ} 1,2017$, La Littérature et le mal, pp. 229-247 ; «Stratégies de désaliénation, déficit émancipatoire et modélisation d'un monde alternatif dans Trop de soleil tue l'amour de Mongo Beti », in Adama Samaké (ed), Genèse, formes et enjeux de l'émancipation dans l'écriture de Mongo Beti, Paris, Publibook, 2017, pp. 93-116 ; "Le Briseur de rosée d'Edwige Danticat à l'épreuve du duvaliérisme : entre exorcisation, quête identitaire, devoir de mémoire et postulation éthique de l'être ensemble ", in Revue LEGS et LITTERATURE, Haït, Legs édition, nº ${ }^{\circ}$, 2017, pp. 183-199.

Co-auteur de Le genre dans tous ses états. Perspectives africaines (Connaissances et savoirs, Paris, 2017), il est également l'auteur de La Société politique africaine postcoloniale chez Francis Bebey (EUE, 2017) et du recueil de poèmes Trajectoires cinquantenaires (EUE, 2017).

Date de réception : 15/05/2019

Date d'acceptation : 22/07/2019 